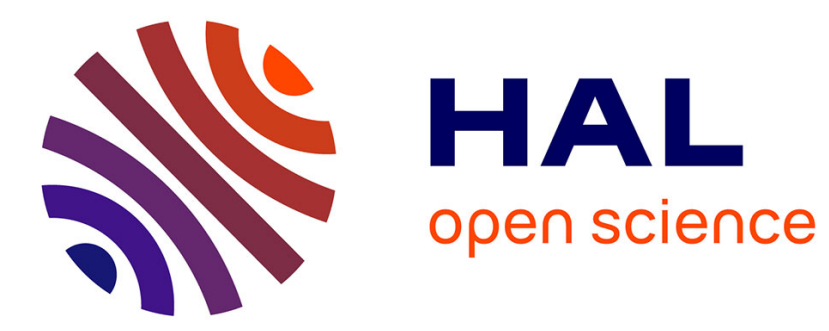

\title{
FRET versus PET: ratiometric chemosensors assembled from naphthalimide dyes and crown ethers
}

\author{
Pavel A. Panchenko, Yuri V. Fedorov, Olga A. Fedorova, Gediminas
}

Jonusauskas

\section{- To cite this version: \\ Pavel A. Panchenko, Yuri V. Fedorov, Olga A. Fedorova, Gediminas Jonusauskas. FRET versus PET: ratiometric chemosensors assembled from naphthalimide dyes and crown ethers. Physical Chemistry Chemical Physics, 2015, 17 (35), pp.22457-23282. 10.1039/C5CP03510D . hal-01199028}

\section{HAL Id: hal-01199028 \\ https://hal.science/hal-01199028}

Submitted on 14 Sep 2015

HAL is a multi-disciplinary open access archive for the deposit and dissemination of scientific research documents, whether they are published or not. The documents may come from teaching and research institutions in France or abroad, or from public or private research centers.
L'archive ouverte pluridisciplinaire HAL, est destinée au dépôt et à la diffusion de documents scientifiques de niveau recherche, publiés ou non, émanant des établissements d'enseignement et de recherche français ou étrangers, des laboratoires publics ou privés.

\section{(c)(1)}

Distributed under a Creative Commons Attribution| 4.0 International License 


\title{
FRET versus PET: ratiometric chemosensors assembled from naphthalimide dyes and crown ethers $\dagger$
}

\author{
Pavel A. Panchenko, ${ }^{a b}$ Yuri V. Fedorov, ${ }^{a}$ Olga A. Fedorova ${ }^{a b}$ and Gediminas Jonusauskas ${ }^{c}$
}

\begin{abstract}
Novel bi-chromophoric naphthalimide derivatives containing benzo-15-crown-5 and N-phenyl-aza-15-crown-5 receptor moieties BNI2 and BNI3 were designed and prepared. Significant Fo"rster resonance energy transfer (FRET) from donor (D) amidonaphthalimide to acceptor (A) amino-naphthalimide chromophores as well as photoinduced electron transfer (PET) between the $N$ aryl receptor and amido-naphthalimide fragment was revealed by the steady-state and time-resolved UV/Vis absorption and fluorescence spectroscopy. Upon the addition of alkaline-earth metal perchlorates to an acetonitrile solution of ligands, FRET mediated fluorescence enhancement was observed, which was a result of inhibition of the PET competitive deactivation pathway. The studied compounds provide an opportunity to register a two-channel fluorescence response upon selective excitation of either of the photoactive units and, thus, might be of interest as ratiometric probes.
\end{abstract}

\section{Introduction}

Förster resonance energy transfer is a unique process making possible the generation of fluorescence signals sensitive to molecular conformation, association and separation in the 1-10 $\mathrm{nm}$ range. ${ }^{1,2}$ This mechanism has been widely used in medicinal diagnostics, optical imaging and molecular biology as a spectroscopic ruler to study the structure of proteins and nucleic acids. In recent years, significant emphasis has been placed on the development of highly selective fluorescent FRET based chemosensors for metal cations because of their potential applications in biochemistry and environmental research. Among various photoinduced processes that are commonly involved in the signaling or response phenomena, the resonance energy transfer seems to be an optimal strategy for designing ratiometric probes. ${ }^{3,4}$

According to the ratiometric method, the analyte concentration can be quantified by using the ratio of intensities of the well resolved fluorescence peaks with reasonable intensities at

\footnotetext{
${ }^{a}$ A. N. Nesmeyanov Institute of Organoelement Compounds of Russian Academy of Sciences (INEOS RAS), 119991, Vavilova str. 28, Moscow, Russia. E mail: pavel@ineos.ac.ru; Fax: +7 49913550 85; Tel: +7 4991358098

${ }^{b}$ D. Mendeleev University of Chemical Technology of Russia,

125047, Miusskaya sqr. 9, Moscow, Russia

${ }^{c}$ Laboratoire Ondes et Matière d'Aquitaine (LOMA), UMR CNRS 5798, Bordeaux University, 33405, 351 Cours de la Libération, Talence, France $\dagger$ Electronic supplementary information (ESI) available. See DOI: 10.1039/ c5cp03510d
}

two different wavelengths for analyte free and analyte bound probes. ${ }^{5}$ Such self-calibration using two emission bands can eliminate the influence of the indicator dye concentration, environmental conditions and instrumental efficiency. Furthermore, the pseudo-Stokes shifts of FRET based probes are larger than the Stokes shifts of either the donor or acceptor dyes; thus, the possible self-quenching as well as fluorescence detection errors due to back scattering effects from the excitation source will be efficiently avoided. ${ }^{6}$

Naphthalimide derivatives are a special class of environmentally sensitive fluorophores. The fluorescence of 1,8-naphthalimides with electron donating groups at the $\mathrm{C}-4$ position of the naphthalene ring has been of great interest for several decades in connection with an array of technical, medical and electronic applications. Because of its intense fluorescence and good photostability, this type of compounds has found application in a number of areas including coloration of polymers, ${ }^{7,8}$ laser active media, ${ }^{9,10}$ fluorescent markers in biology, ${ }^{11-13}$ anticancer agents and analgesics in medicine, ${ }^{14}$ electroluminescent materials, ${ }^{15-17}$ fluorescence switchers, ${ }^{18-20}$ liquid crystal displays ${ }^{21,22}$ and ion probes. ${ }^{23,24}$

To date, some examples of naphthalimide based FRET probes have been reported in the literature. Georgiev et al. described the synthesis and $\mathrm{pH}$-sensitive fluorescence of the PAMAM dendron core and peripherally functionalized it with amino- and alkoxynaphthalimides as donor and acceptor fluorophores. ${ }^{25-27} \mathrm{~A}$ FRET-based ratiometric chemosensor for in vitro cellular fluorescence analyses of $\mathrm{pH}$ based on a naphthalimide-coumarin 
system was reported by Zhou et $a .^{28}$ Selective ratiometric chemosensors for $\mathrm{Cu}^{2+}$ and $\mathrm{Zn}^{2+}$ were obtained using dansylamidenaphthalimide conjugates with variable polymethylene linker lengths between the chromophores. ${ }^{29,30} \mathrm{~A}$ few chemosensors for metal cations and protons containing amino-naphthalimide and rhodamine units in which the spirolactam to ring-open amide equilibrium of rhodamine dyes is used to switch FRET from the amino-naphthalimide fragment were studied. ${ }^{31-40}$

We have recently developed mono-chromophoric aminoand amido-naphthalimide derivatives MNI4-6 (Scheme 1) bearing crown ether groups as fluorescent sensors exploiting the photoinduced electron transfer process. ${ }^{41-43}$ These compounds displayed pronounced enhancement of emission intensity by coordination with metal cations, which was a result of inhibition of PET between the crown ether receptor conjugated with the $N$-phenyl ring and the fluorophore. Herein, we report the design, synthesis and investigation of cation-dependent behavior of FRET-based ratiometric sensors BNI2-3 by integrating amido-naphthalimide probes MNI5 and MNI7 as FRET donors and amino-naphthalimide MNI1 as an FRET acceptor. In this case, the strategy for detection of metal ions is based on modulating the FRET process, and thus emission intensity of the acceptor aminonaphthalimide fragment, by means of incorporation of a competitive PET deactivation pathway. In order to receive a more complete comparative picture of the influence of crown ether groups on the efficiency of FRET interaction in a bi-chromophoric system we prepared non-ionophoric dyad compound BNI1. Naphthalimides MNI1, ${ }^{44}$ MNI2, ${ }^{44}$ MNI3, ${ }^{44}$ MNI5 $^{44}$ and MNI7 $^{43}$ have been synthesized earlier and were also included in photophysical studies as reference compounds.

\section{Experimental section}

\section{Steady-state optical measurements}

The absorption spectra were taken on a Varian-Cary 5G spectrophotometer. The fluorescence quantum yield measurements were performed using a Varian-Cary 5G spectrophotometer and a FluoroMax-3 spectrofluorometer. Spectral measurements were carried out in air-saturated acetonitrile solutions (acetonitrile of spectrophotometric grade, water content $<0.005 \%$, Aldrich) at $20 \pm 1{ }^{\circ} \mathrm{C}$; the concentrations of the studied compounds were about $0.5-2.0 \times 10^{-5} \mathrm{M}$. All measured fluorescence spectra were corrected for nonuniformity of detector spectral sensitivity. Coumarin 481 in acetonitrile $\left(\varphi^{\mathrm{fl}}=0.08\right)^{45}$ was used as a reference for the fluorescence quantum yield measurements. The fluorescence quantum yields were calculated using ${ }^{46}$

$$
\varphi_{\mathrm{i}}^{\mathrm{fl}}=\varphi_{0}^{\mathrm{fl}} \frac{S_{\mathrm{i}}(1}{S_{0}\left(10^{-A_{0}}\right) n_{\mathrm{i}}{ }^{2}}
$$

where $\varphi_{\mathrm{i}}^{\mathrm{fl}}$ and $\varphi_{0}^{\mathrm{fl}}$ are the fluorescence quantum yields of the studied solution and the standard compound, respectively; $A_{\mathrm{i}}$ and $A_{0}$ are the absorptions of the studied solution and the standard, respectively; $S_{\mathrm{i}}$ and $S_{0}$ are the areas underneath the curves of the fluorescence spectra of the studied solution and the standard, respectively; and $n_{\mathrm{i}}$ and $n_{0}$ are the refraction indices of the solvents for the substance under study and the standard compound $\left(n_{\mathrm{i}}=n_{0}=1.342\right.$, acetonitrile).

\section{Time-resolved fluorescence setup}

A Ti:sapphire laser system emitting pulses of $0.6 \mathrm{~mJ}$ and $30 \mathrm{fs}$ at $800 \mathrm{~nm}$ and a $1 \mathrm{kHz}$ pulse repetition rate (Femtopower Compact Pro) with a home-built optical parametric generator and frequency mixers was used to excite the samples at the maximum of the steady-state absorption band. All excited-state lifetimes were obtained by using depolarized excitation light. The highest pulse energies used to excite fluorescence did not exceed $100 \mathrm{~nJ}$ and the average power of the excitation beam was $0.1 \mathrm{~mW}$ at a pulse repetition rate of $1 \mathrm{kHz}$ focused onto a spot with a diameter of $0.1 \mathrm{~mm}$ in the $10 \mathrm{~mm}$-long fused-silica cell. The fluorescence emitted in the forward direction was collected by reflective optics and focused with a spherical mirror onto the input slit of a spectrograph (Chromex 250) coupled to a streak camera (Hamamatsu 5680 equipped with a fast single sweep unit M5676, temporal resolution $2 \mathrm{ps}$ ). Convolution of a rectangular streak camera slit in the sweep range of 250 ps with electronic jitter of the streak camera trigger pulse provided a Gaussian (over four decades) temporal apparatus function with a full width at half-maximum of $20 \mathrm{ps}$. The fluorescence<smiles>Nc1ccc2c3c(cccc13)C(=O)N(c1ccc(CC(=O)Nc3ccc4c5c(cccc35)C(=O)N([Al])C4)cc1)C2=O</smiles><smiles>[R]Nc1ccc2c3c(cccc13)C(=O)N([Al])C2=O</smiles>
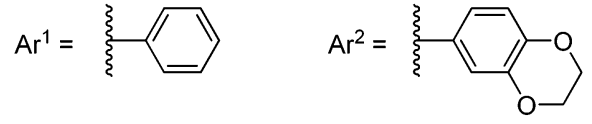

$\mathrm{Ar}^{3}=$<smiles>CC(C)(C)c1ccc2c(c1)OCCOCCOCCOCCO2</smiles><smiles>C[C@@H]([Al])c1ccc(N2CCOCCOCCOCCOCC2)cc1</smiles>

Scheme 1 Structure of compounds MNI1-7 and BNI1-3. 
kinetics were later fitted by means of the Levenberg-Marquardt least-squares curve-fitting method using a solution of the differential equation describing the evolution in time of a single excited state and neglecting depopulation of the ground state according to

$$
\frac{\mathrm{d} I}{\mathrm{~d} t}=\operatorname{Gauss}\left(t_{0}, \Delta t, A\right) \quad \frac{I(t)}{\tau}
$$

where $I(t)$ is the fluorescence intensity, Gauss is the Gaussian profile of the excitation pulse, in which $t_{0}$ is the excitation pulse arrival delay, $\Delta t$ is the excitation pulse width, and $A$ is the amplitude. The parameter $\tau$ is the lifetime of the excited state. The initial condition for the equation is $I(\infty)=0$. Typically, the fit shows a $\chi^{2}$ value (Pirson's criteria) better than $10^{-4}$ and a correlation coefficient $R>0.999$. The uncertainty of the lifetime was better than $1 \%$. Routinely, the fluorescence accumulation time in our measurements did not exceed $90 \mathrm{~s}$.

\section{Transient absorption setup}

The laser system and frequency-conversion apparatus employed to excite samples were the same as for time-resolved fluorescence measurements. White light continuum (360-1000 nm) pulses generated in a $5 \mathrm{~mm}$ methanol cell were used as the probe. The variable delay time between excitation and probe pulses was obtained by using a delay line with $0.1 \mathrm{~mm}$ resolution. The solutions were placed in a $1 \mathrm{~mm}$ circulating cell. The white light signal and reference spectra were recorded using a two-channel fiber spectrometer (Avantes Avaspec-2048-2). A home-written acquisition and experiment-control program in LabView made it possible to record transient spectra with an average error of less than $10^{-4}$ times the optical density at all wavelengths. The temporal resolution of our setup was better than 60 fs. Temporal chirp of the probe pulse was corrected using a computer program with respect to a Lawrencian fit of a Kerr signal generated in a $0.2 \mathrm{~mm}$ glass plate used in place of the sample.

\section{Equilibrium constant determination}

Complex formation of compounds BNI2 and BNI3 with $\mathrm{Mg}^{2+}$ and $\mathrm{Ca}^{2+}$ in acetonitrile at $20 \pm 1{ }^{\circ} \mathrm{C}$ was studied by spectrofluorometric titration. ${ }^{47,48}$ The ratio of the dye to $\mathrm{M}^{2+}$ was varied by adding aliquots of a solution of metal perchlorate $\$$ of known concentration to a solution of ligand BNI2 or BNI3 of known concentration. The fluorescence spectrum of each solution was recorded, and the stability constants of the complexes were determined using the SPECFIT/32 program (Spectrum Software Associates, West Marlborough, MA). The following equilibria were considered in the fitting (eqn (3) and (4), L = BNI2 or BNI3; $\mathrm{M}^{2+}=\mathrm{Mg}^{2+}$ or $\left.\mathrm{Ca}^{2+}\right)$ :

$$
\begin{gathered}
\mathrm{L}+\mathrm{M}^{2+} \rightleftarrows(\mathrm{L}) \cdot \mathrm{M}^{2+} \\
2 \mathrm{~L}+\mathrm{M}^{2+} \rightleftarrows(\mathrm{L})_{2} \cdot \mathrm{M}^{2+}
\end{gathered}
$$

‡ Calcium perchlorate for complexation studies was dried in vacuum (7 $8 \mathrm{~mm} \mathrm{Hg}$ ) at $240{ }^{\circ} \mathrm{C}$ and kept anhydrous over $\mathrm{P}_{2} \mathrm{O}_{5}$ in a desiccator (Caution! Calcium perchlorate may explode while heating. It decomposes at $270{ }^{\circ} \mathrm{C}^{49}$ ). Anhydrous magnesium perchlorate was used as received.
In doing so, it was found that the experimental data corresponded to the theoretical ones if only eqn (3) was taken into account and the formation of the complexes with a composition of $2: 1$ was not observed.

The equilibrium constants for protonation of ligand BNI3 was not determined by this method because of high stability $\left(K>10^{7} \mathrm{M}^{-1}\right)$ of the protonated form $(\mathrm{BNI} 3) \cdot \mathrm{H}^{+}$.

\section{Determination of fluorescence quantum yields of complexes}

The fluorescence quantum yields of complexes (BNI2) $\cdot \mathrm{Mg}^{2+}$ and (BNI3) $\mathrm{Ca}^{2+}$ were determined using solutions of ligands BNI2-3 in $\mathrm{CH}_{3} \mathrm{CN}$ containing an excess of the corresponding metal perchlorate in order to obtain $90-95 \%$ of ligand bound with the cation. The required $\mathrm{M}\left(\mathrm{ClO}_{4}\right)_{2}$ excess was calculated from the known stability constants using the SPECFIT/32 program. The measurement of $\varphi^{\mathrm{fl}}$ for (BNI3) $\cdot \mathrm{H}^{+}$was done in the presence of 2 eq. $\mathrm{HClO}_{4}$ in ligand solutions, which can be understood from the fact that further addition of $\mathrm{HClO}_{4}$ did not result in the fluorescence enhancement and complex formation had already been completed.

\section{Results and discussion}

\subsection{Design and synthesis of the compounds}

Following the classical description of a FRET model, a requirement for efficient energy transfer is that there should be a spectral overlap between the emission of the donor and absorbance of the acceptor dyes. It is well known that absorption and fluorescence characteristics of 1,8-naphthalimides depend on the nature of the substituent at the C-4 position of the 1,8-naphthalimide ring involved in the charge transfer interaction with the dicarboximide moiety. In the construction of dyad probes BNI2-3, amidonaphthalimide was chosen as an energy donor, because it has strong emission in the visible range centered at 440-460 nm, which covers a part of amino-naphthalimide absorption $\left(\lambda_{\max }=\right.$ 410-430 nm). ${ }^{44}$ Fig. 1 shows the overlap between the absorption

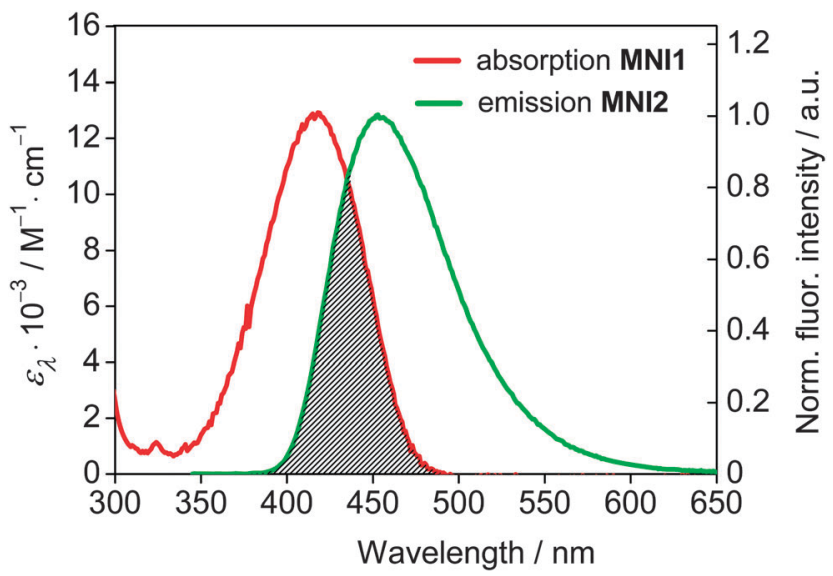

Fig. 1 Overlap between the MNI1 absorption and MNI2 emission spectra in acetonitrile. The concentration of both compounds is $5.0 \times 10^{6} \mathrm{M}$. Excitation wavelength $\lambda_{\mathrm{ex}}=340 \mathrm{~nm}$. 
and fluorescence spectra of reference compounds MNI1 and MNI2 in acetonitrile, fulfilling a favorable condition for FRET.

Another factor, which influences the FRET efficiency, is the space separation of donor (D) and acceptor (A) units. Since the transfer rate drops rapidly with the increase of the D-A distance, we used a rather short and rigid phenyl spacer in BNI2-3. Furthermore, low conformational flexibility of the phenyl group would also hinder a dyad molecule from adopting a conformation where both naphthalimide moieties are arranged as an internal aggregate stabilized by $\pi$-stacking interaction, in which the formation of non-emissive state could be suggested. Crown ether groups were incorporated in the $N$-aryl fragment of a more electron deficient amido-naphthalimide chromophore (in comparison with amino-naphthalimide), because in this case a strong PET interaction is expected for both benzo-15crown-5 and $N$-phenylaza-15-crown-5 ether receptors. ${ }^{41,43}$

The synthesis of bi-chromophoric naphthalimide derivatives BNI1-3 was carried out using the convergent scheme. Starting from 4-nitronaphthalic anhydride 1, the intermediate 4-nitro1,8-napthalimide 3 was afforded by a reaction with (4-aminophenyl)acetic acid in AcOH media (Scheme 2). Compound 3 was refluxed with thionyl chloride to produce chloroanhydride $\mathbf{4}$, which further was stirred together with preliminary synthesized amino-naphthalimides MNI1, MNI4 and MNI6 in anhydrous toluene or 1,4-dioxane. At the final step, acylation products 5a-c were subjected to reduction using tin(II) chloride in the presence of hydrochloric acid. The experimental details concerning the synthesis of target compounds can be found in the ESI. $\dagger$

\subsection{Photophysical properties of the compounds}

The photophysical characteristics of BNI1-3 were measured in acetonitrile solution and the data are presented in Table 1. First of all, we studied the resonance energy transfer characteristics of the non-crowned derivative BNI1. The absorption spectrum of BNI1 (Fig. 2a), as expected, shows the presence of two long wavelength bands corresponding to the absorption location of the amido-naphthalimide donor (reference compound MNI2) and the amino-naphthalimide acceptor (reference compound MNI1). Given a high level of additivity of the BNI1 spectrum, there could be a lack of any overlap of molecular orbitals between the individual fluorophores in the BNI1 ground state.

Selective excitation of BNI using $340 \mathrm{~nm}$ light produced a single emission band at $520 \mathrm{~nm}$ (Fig. 2b), which is characteristic of<smiles>CC(C)CC(CC(=O)O)C(=O)O</smiles>

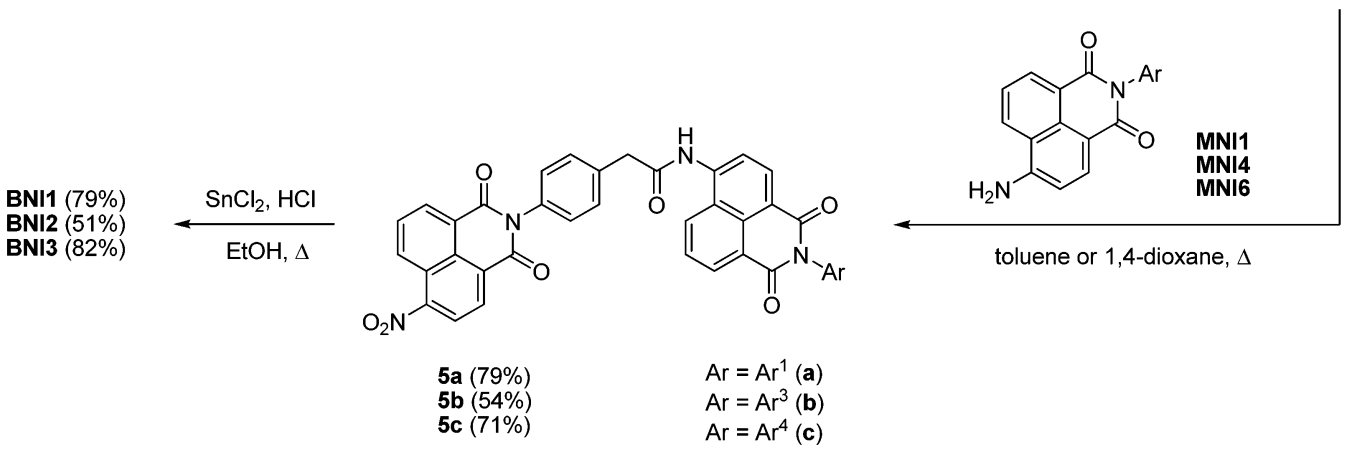

Scheme 2 Synthetic route to compounds BNI1-3.

Table 1 Photophysical properties and stability constants of mono and bi chromophoric naphthalimides and their complexes in acetonitrile at $20{ }^{\circ} \mathrm{C}$

\begin{tabular}{llllll}
\hline Compound & $\lambda_{\text {max }}^{\text {abs }} / \mathrm{nm}$ & $\varepsilon_{\lambda} \times 10^{3} / \mathrm{M}^{1} \mathrm{~cm}^{1}$ & $\lambda_{\text {max }}^{\mathrm{fl}}\left(\lambda_{\mathrm{ex}}\right) / \mathrm{nm}$ & $\varphi^{\mathrm{fl}}$ & $\Phi_{\text {FRET }}$ \\
\hline MNI1 & 417 & 12.9 & $518(420)$ & 0.55 & \\
MNI2 & 367 & 17.3 & $454(365)$ & 0.90 & \\
MNI3 & 366 & 17.0 & $454(365)$ & 0.0048 & \\
MNI5 & 366 & 17.4 & $454(365)$ & 0.0030 & 0.99997 \\
MNI7 & 366 & 15.9 & $456(365)$ & 0.0017 & 0.64 \\
BNI1 & 371 & 20.2 & $520(340)$ & 0.47 & 0.34 \\
BNI2 & 368 & 16.2 & $520(340)$ & 0.28 & $5.69 \pm 0.03$ \\
(BNI2) $\mathrm{Mg}^{2+}$ & 370 & 16.9 & $520(340)$ & 0.50 & \\
BNI3 & 368 & 16.3 & $519(340)$ & 0.059 & $5.04 \pm 0.01$ \\
(BNI3) $\mathrm{Ca}^{2+}$ & 372 & 16.6 & $520(340)$ & 0.36 & \\
(BNI3) $\mathrm{H}^{+}$ & 372 & 15.1 & $520(340)$ & 0.45 & Not determined
\end{tabular}

${ }^{a}$ The dimension of $K$ is $\mathbf{M}^{1}$. 

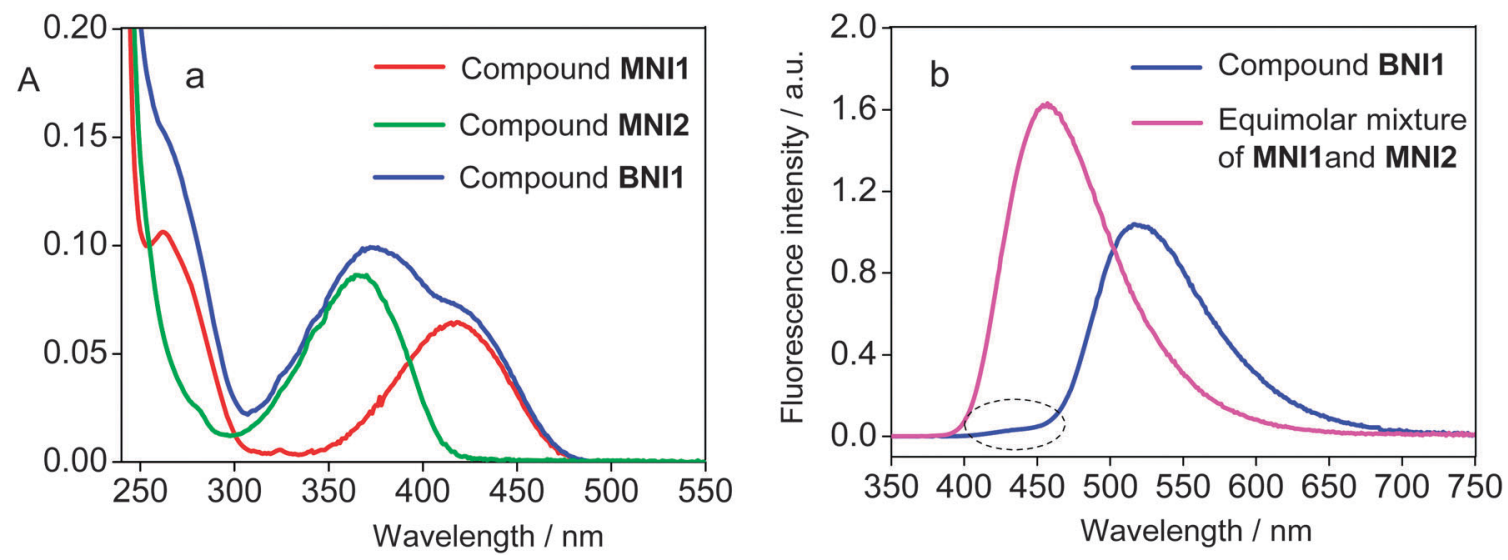

Fig. 2 Absorption spectra of MNI1, MNI2 and BNI1 (a) and fluorescence spectra of BNI1 and an equimolar mixture of fluorophores MNI1 and MNI2 (b) in acetonitrile. The concentration of all compounds is $5.0 \times 10^{6} \mathrm{M}$. Excitation wavelength $\lambda_{\mathrm{ex}}=340 \mathrm{~nm}$.

the amino-naphthalimide fragment.§ In contrast, under the same conditions, an equimolar mixture of fluorophores MNI1 and MNI2 demonstrated emission at around $450 \mathrm{~nm}$ originating from amido-naphthalimide MNI2. This result indicates that in a bi-chromophoric system the excitation energy transfers effectively from donor to acceptor units, whereas in the case of a dilute solution of an equimolar mixture FRET interaction is not observed.

To get deeper insight into the nature of excited state deactivation pathways we measured the excited state lifetime of the donor chromophore in the compound BNI1. In comparison with a single amido-naphthalimide MNI2 ( $\tau=10 \mathrm{~ns})$, it was shorter by more than four orders of magnitude $\left(\tau_{\mathrm{D}}=0.31 \mathrm{ps}\right)$, $\uparrow$ implying the existence of a fast non-radiative process more likely to be the resonance energy transfer. The efficiency of the energy transfer $\left(\Phi_{\text {FRET }}\right)$ in dyad compound BNI1 was calculated to be 0.99997 $(99.997 \%)$ according to ${ }^{2}$

$$
\Phi_{\mathrm{FRET}}=1 \quad \frac{\tau_{\mathrm{D}}}{\tau}
$$

A pretty close value of $\Phi_{\text {FRET }}(99.95 \%)$ was obtained from calculations using Förster theory (for details see the ESI $\dagger$ ). Such a high value of $\Phi_{\text {FRET }}$ could be a result of a rather short distance between donor and acceptor chromophores $(r=12.0 \AA$ as obtained from the optimized geometry of BNI1 (Fig. S1, ESI $\dagger$ )), which is about 3.5 times shorter compared to the critical Förster radius $\left(R_{0}=41.8 \AA\right)$ for this system.

The introduction of crown ether substituents in the naphthalimide dyad molecule BNI1 results in the reduction of the energy transfer efficiency. As it was shown in our previous publications, the presence of electron releasing benzo-15-crown-5, benzo-1,4dioxane or $N$-phenyl-aza-15-crown-5 ether groups in the $N$-aryl fragment of amido-naphthalimides MNI3, MNI5 and MNI7 leads

$\S$ A weak shoulder at $450 \mathrm{~nm}$ in the fluorescence spectrum of BNI1 results from residual fluorescence of the donor chromophore. The appearance of this shoulder can be explained by the relatively high fluorescence quantum yield of MNI2 (Table 1).

T The data for MNI2 are obtained from the analysis of fluorescence kinetics, for compound BNI1 from the analysis of transient absorption spectra (see the Experimental section). to a dramatic decrease of emission intensity with respect to the highly emissive non-crowned derivative MNI2 due to efficient photoinduced electron transfer between the naphthalimide chromophore and receptor moieties. ${ }^{41,43,44}$ Keeping this in mind, one could conclude that in the case of crown-containing dyad compounds BNI2 and BNI3, the deactivation of the donor chromophore excited state would proceed via both electron and energy transfer. Additionally, radiative decay (fluorescence) and other possible non-radiative ways of relaxation (except for PET and FRET) should be taken into consideration. A representative scheme showing all these energy degradation channels in dyads BNI2 and BNI3 is depicted in Fig. 3. Provided each photophysical process in Fig. 3 is characterized by the first order rate constant, the $\Phi_{\mathrm{FRET}}$ value can be expressed as the ratio of the FRET rate constant $\left(k_{\mathrm{FRET}}\right)$ to the sum of rate constants of all other processes mentioned above (eqn (6)):

$$
\Phi_{\mathrm{FRET}}=\frac{k_{\mathrm{FRET}}}{k_{\mathrm{FRET}}+k_{\mathrm{PET}}+k_{\mathrm{R}}+k_{\mathrm{NR}}}
$$

In eqn (6), $k_{\mathrm{R}}$ is the radiative rate constant of the amidonaphthalimide chromophore, and $k_{\mathrm{NR}}$ describes its nonradiative relaxation which is not related to energy or electron transfer. To estimate the sum $\left(k_{\mathrm{R}}+k_{\mathrm{NR}}\right)$ we used the value inversely proportional to the fluorescence lifetime of compound MNI2, where FRET and PET channels are not realized (eqn (7)). $k_{\mathrm{FRET}}$ was calculated as the difference between deactivation rate constants for compounds BNI1 and MNI2, supposing that the decrease of the excited state lifetime going from MNI2 to BNI1 is only a result of FRET interaction (eqn (8)):

$$
\begin{aligned}
k_{\mathrm{R}}+k_{\mathrm{NR}}=\frac{1}{\tau}=\frac{1}{10.2 \times 10^{-9}}=9.8 \times 10^{7} \mathrm{~s}^{-1} \\
\begin{aligned}
k_{\mathrm{FRET}} & =\frac{1}{\tau_{\mathrm{D}}} \quad \frac{1}{\tau}=\frac{1}{0.31 \times 10^{-12}} \quad \frac{1}{10.2 \times 10^{-9}} \\
& =3.2 \times 10^{12} \mathrm{~s}^{-1}
\end{aligned}
\end{aligned}
$$

Simple analysis of eqn (6) clearly shows that the FRET efficiency in a bi-chromophoric system can be modulated by 


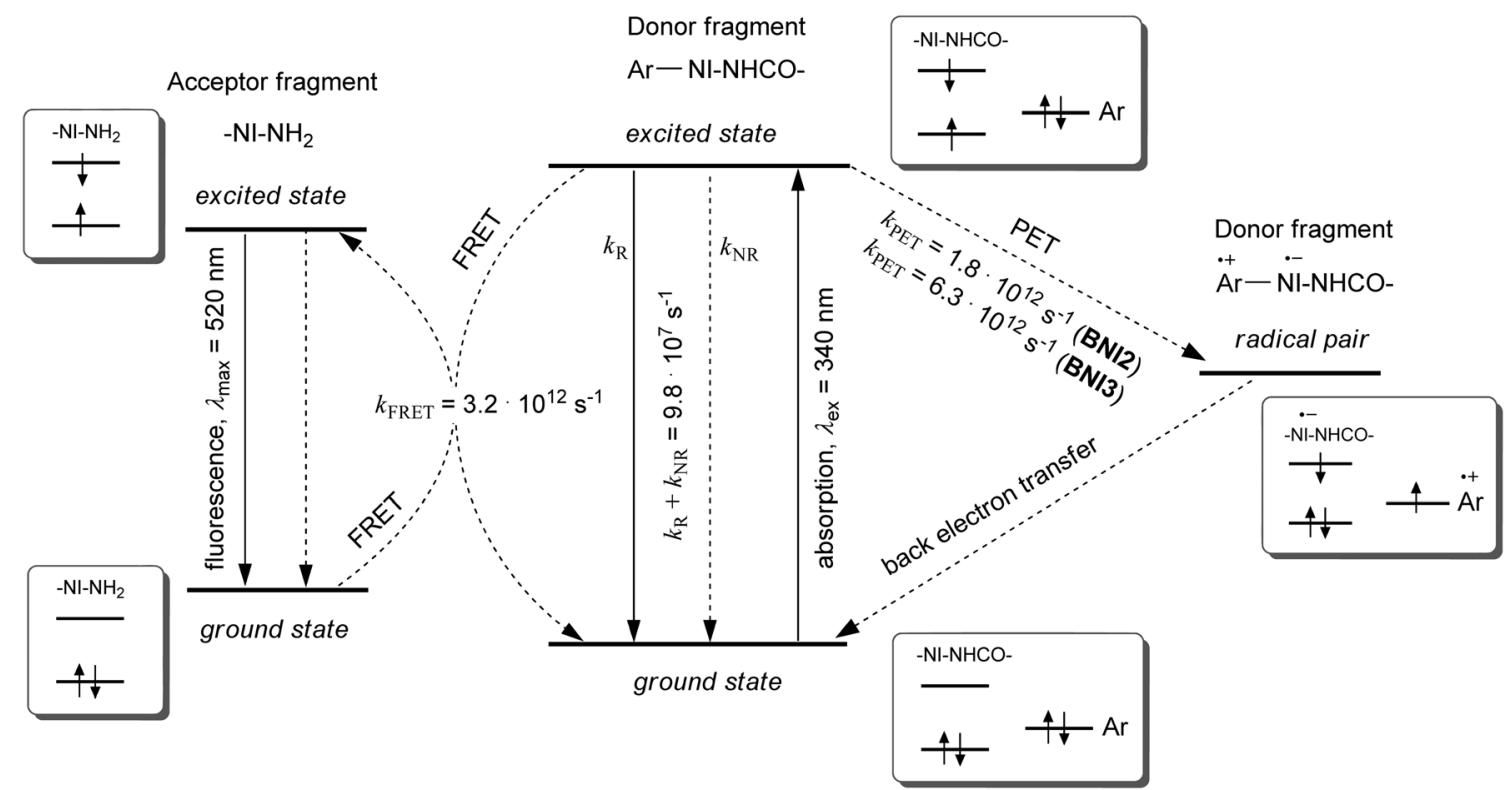

Fig. 3 Excited state relaxation pathways of donor and acceptor chromophores in crown containing dyads BNI2 and BNI3. Plain and dashed arrows denote radiative and non radiative processes, respectively. "NI" denotes the naphthalimide moiety.

changing the rate of photoinduced electron transfer. Thus, the increase of PET donor ability would reduce the amount of energy transferred to the amino-naphthalimide acceptor and thereby quench the fluorescent output signal. For the evaluation of $k_{\mathrm{PET}}$ in dyads BNI2 and BNI3, we used relaxation kinetics data of amido-naphthalimides MNI3 and MNI7 in which PET is the main deactivation pathway. As an example, Fig. 4a shows the transient absorption spectra of MNI3 at different time delays between pump and probe pulses. It can be seen that the relaxation of singlet excited state $\left(\mathrm{S}_{1}\right)$ proceeds with a concomitant growth of two novel bands probably corresponding to the ion-radical intermediates. An intense signal with a maximum at $426 \mathrm{~nm}$ was assigned to benzodioxane cation-radical absorption as it falls in the wavelength interval $400-480 \mathrm{~nm}$ where the characteristic bands of either isomeric dimethoxybenzenes ${ }^{50}$ or $N, N$-dimethylaniline cation-radicals ${ }^{51}$ are located. From the analysis of kinetic data (Fig. $4 \mathrm{~b}$ ), the PET rate constant $\left(k_{\mathrm{PET}}\right)$ for compound MNI3 was found to be as high as $1.8 \times 10^{12} \mathrm{~s}^{-1}$. Similar changes were observed in the transient absorption spectrum of MNI7 resulting in a $k_{\mathrm{PET}}$ value of $6.3 \times 10^{12} \mathrm{~s}^{-1}$.

As the comparison of $k_{\mathrm{PET}}$ and $k_{\mathrm{FRET}}$ shows, PET and FRET are comparably competing processes. Using the data of timeresolved experiments and eqn (6), we found that introduction of the benzo-15-crown-5 ether receptor into the $N$-phenyl ring of BNI1 decreases the energy transfer efficiency from $99.997 \%$ to $64 \%$, whereas the presence of the aza-15-crown-5 ether group possessing a more strong PET donor ability in compound BNI3 results in only $34 \%$ of excitation energy involved in FRET.
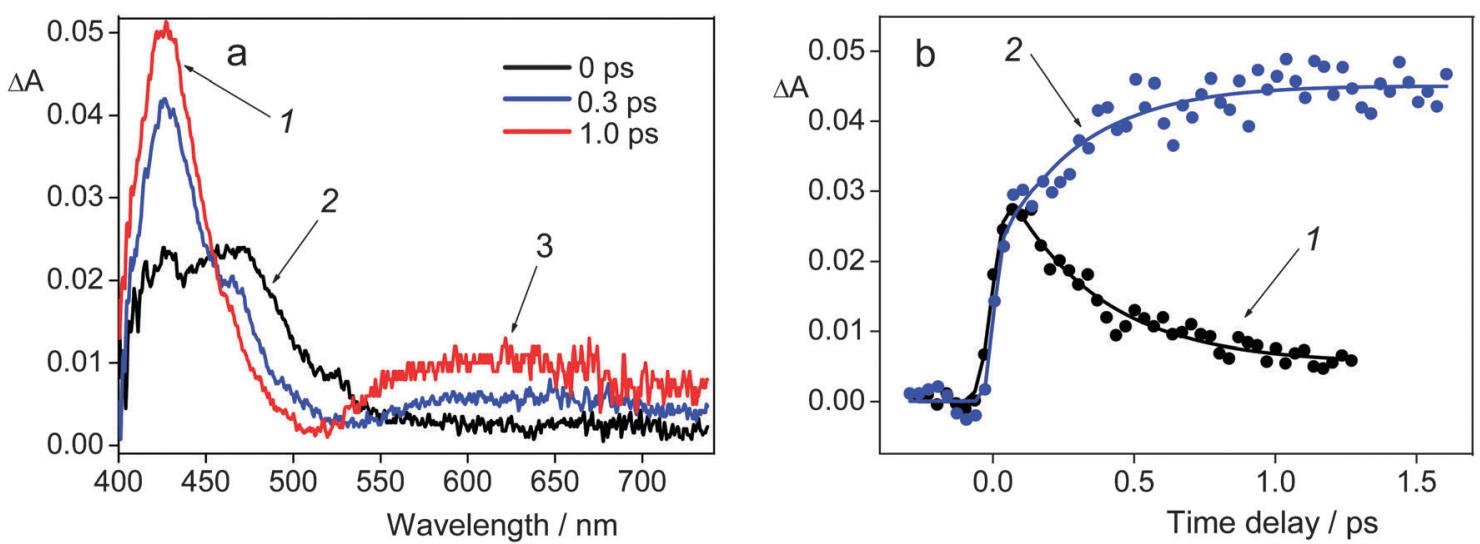

Fig. 4 Transient absorption spectra of MNI3 at different time delays between pump and probe pulses (a) and transient absorption time profile of MNI3 (b) in acetonitrile. (a) 1 Absorption band corresponding to the cation radical of the benzodioxane fragment; 2 absorption of the singlet $\mathrm{S}_{1}$ state of the amido naphthalimide chromophore; 3 the possible position of the absorption signal of the amido naphthalimide anion radical. (b) 1 Time profile at the absorption maximum of the amido naphthalimide excited $S_{1}$ state $(480 \mathrm{~nm}) ; 2$ Time profile at the absorption maximum of the benzodioxane cation radical $(420 \mathrm{~nm})$. 


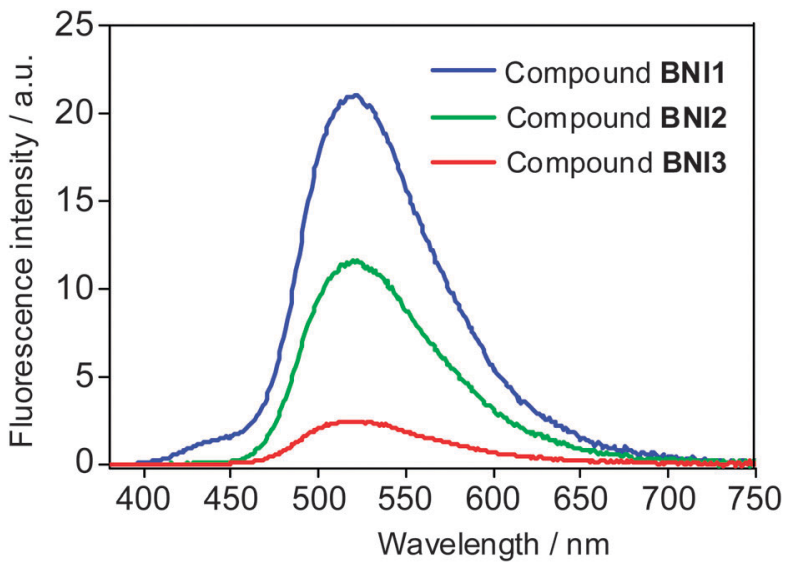

Fig. 5 Fluorescence spectra of compounds BNI1-3 in acetonitrile. The concentration of all compounds is $6.5 \times 10^{6} \mathrm{M}$. Excitation wavelength $\lambda_{\mathrm{ex}}=340 \mathrm{~nm}$.

The changes in the $\Phi_{\text {FRET }}$ value in the range of naphthalimide dyads BNI1-BNI2-BNI3 was found to be in full agreement with the steady-state optical data showing the reduction of the fluorescence intensity and fluorescence quantum yield for the crown-containing compounds (Fig. 5, Table 1).

\subsection{Complex formation of naphthalimide dyads}

We further examined the ability of crown-containing dyads BNI2 and BNI3 to switch their photophysical characteristics as a result of metal ion binding. For the complexation experiments we chose $\mathrm{Mg}^{2+}$ and $\mathrm{Ca}^{2+}$, because these cations are known to form stable complexes with benzo-15-crown-5 and aza-15-crown-5 ethers in an acetonitrile solution, ${ }^{41,43}$ which is very convenient for studying cation-induced optical effects.

The addition of magnesium and calcium perchlorates to the solution of ligands BNI2 and BNI3 in MeCN does not virtually change the position and intensity of the long wavelength absorption bands (compare $\lambda_{\max }^{\mathrm{abs}}$ and $\varepsilon_{\lambda}$ values for the free ligands and corresponding complexes in Table 1). This observation indicates that charge transfer transitions in both naphthalimide units in molecules BNI2 and BNI3 are not affected by the coordination of metal ions with the crown ether receptors. Similar results were obtained for the mono-chromophoric naphthalimides MNI4-7 in our previous publications, ${ }^{41,43}$ where the negligible changes in the absorption spectra were attributed to the lack of conjugation between the $N$-aryl group and naphthalimide chromophore resulting from the nearly orthogonal disposition of these fragments in space. Apparently, the same structural feature persists in dyads BNI2 and BNI3 explaining the similarity in spectral behavior.

In contrast to the absorption spectra, the addition of $\mathrm{Mg}^{2+}$ and $\mathrm{Ca}^{2+}$ led to pronounced changes in the emission intensity. Considering the ability of the PET process to be fully or partially blocked upon the complex formation, one should expect that the binding of metal cations by BNI2 and BNI3 molecules would cause an increase in energy transfer efficiency giving rise to fluorescence enhancement of the acceptor amino-naphthalimide chromophore. As depicted in Fig. 6, the described situation was observed in the experiment. The fluorescence emission spectra of compounds BNI2 and BNI3 were recorded in the presence of graduated amounts of the corresponding metal perchlorates and the titration data were applied for the calculation of stability constants (Table 1).

From the analysis of cation-induced changes in the emission spectra, it can be seen that optical responses for compounds BNI3 and BNI2 are different and, indeed, BNI3 demonstrates a higher extent of fluorescence enhancement. The reason for the observed difference might be explained by the more efficient PET in the free ligand BNI3, which intensifies FRET switching contrast. It should, however, be said that the binding of $\mathrm{Ca}^{2+}$ by BNI3 does not seem to quench PET interaction completely, because the fluorescence quantum yield of complex (BNI3) $\cdot \mathrm{Ca}^{2+}$ $\left(\varphi^{\mathrm{fl}}=0.36\right)$ is somewhat lower compared to that of dyad BNI1 $\left(\varphi^{\mathrm{fl}}=0.47\right)$ not containing any crown ether moiety. Similar results were obtained for complex (MNI7) $\cdot \mathrm{Ca}^{2+}$, where sensor properties of monochromophoric naphthalimides MNI6 and MNI7 were studied. ${ }^{43}$ Another factor approving the residual PET
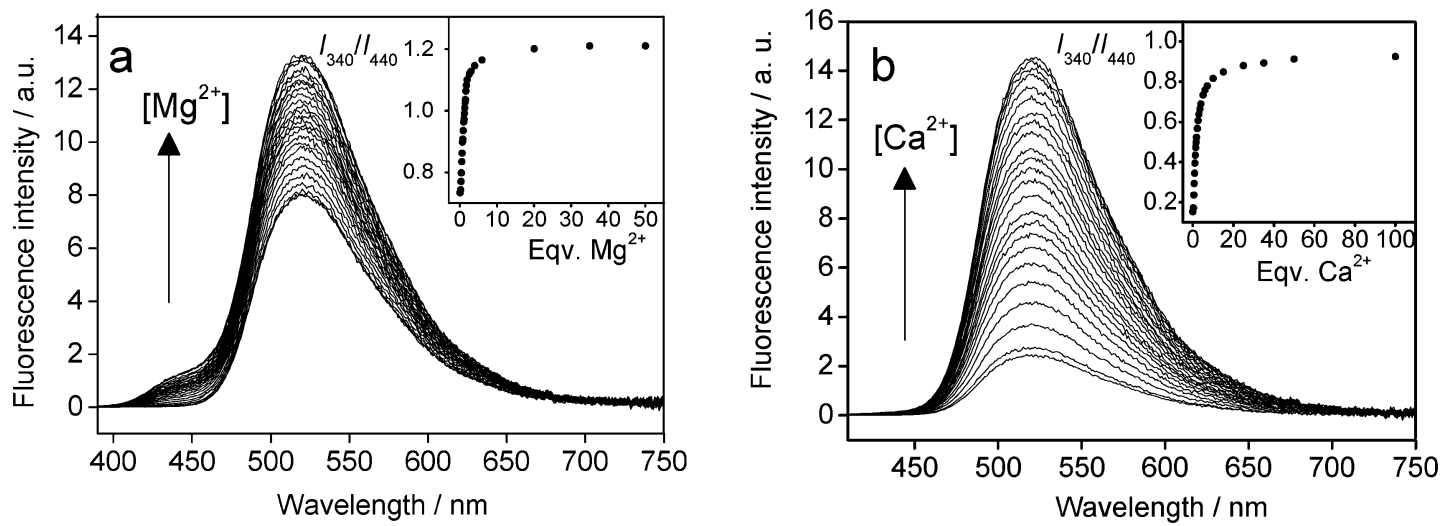

Fig. 6 The changes in the fluorescence spectrum of compounds BNI2 (a) and BNI3 (b) in acetonitrile solution. Excitation wavelength $\lambda_{\text {ex }}=340 \mathrm{~nm}$. The insets show the ratio of the fluorescence intensity at $520 \mathrm{~nm}$ measured using excitation light $\lambda_{\mathrm{ex}}=340 \mathrm{~nm}(/ 340)$ to the fluorescence intensity at $520 \mathrm{~nm}$ measured using excitation light $\lambda_{\mathrm{ex}}=440 \mathrm{~nm}\left(\mathrm{I}_{440}\right)$. (a) The concentration $C_{\mathrm{L}}=4.5 \times 10{ }^{6} \mathrm{M}$ for ligand BNI2; (b) the concentration $C_{\mathrm{L}}=6.5 \times 10{ }^{6} \mathrm{M}$ for ligand BNI3. 
in (BNI3) $\cdot \mathrm{Ca}^{2+}$ is that the emission spectrum of the $\mathrm{Ca}^{2+}$-saturated solution of BNI3 does not contain the short wavelength shoulder arising from the fluorescence of the donor chromophore which, in contrast, emerges in the case of BNI1 (Fig. 2b) and (BNI2) $\cdot \mathbf{M g}^{2+}$ (Fig. 6a).

In order to confirm our assumption concerning partial inhibition of PET in (BNI3) $\cdot \mathrm{Ca}^{2+}$, we measured spectral characteristics of the protonated form of ligand BNI3 (Table 1). The addition of $\mathrm{HClO}_{4}$ in an acetonitrile solution of BNI3 resulted in the formation of highly fluorescent complex (BNI3) $\cdot \mathrm{H}^{+}$with $\varphi^{\mathrm{fl}}$ value similar to that of BNI1. In this complex the lone electron pair of anilino nitrogen of the receptor is fully engaged in coordination with the cation due to the formation of the $\mathrm{N}-\mathrm{H} \sigma$-bond, which breaks the conjugation, significantly lowers the potential energy of the $N$-aryl group and thus completely quenches the PET process.

Finally, we would like to clear how the studied bi-chromophoric compounds can be applied for the ratiometric detection of ions. The main idea of ratiometric measurements is based on self-calibration of a sensor system. This means that probe response should contain not only the signal reporting the analyte binding, but there must be a different signal (or signals) that could allow us to account or compensate all the effects that influence the fluorescence parameter(s) besides the analyteprobe interaction. In the case of crown-containing dyads BNI2 and BNI3, the excitation of the amino-naphthalimide chromophore using visible light $\left(\lambda_{\mathrm{ex}}=440 \mathrm{~nm}\right)$ leads to emission at $520 \mathrm{~nm}$. Noteworthy, this signal does not depend on whether a cation is present in the crown ether moiety or not and, hence, can be used for self-calibration. In contrast, the fluorescence output obtained as a result of donor chromophore excitation with UV light $\left(\lambda_{\mathrm{ex}}=340 \mathrm{~nm}\right)$ is strongly cation-dependent. Thus, the ratio of the yellow-green emission intensities at $520 \mathrm{~nm}$ obtained at $\lambda_{\text {ex }}=340$ and $440 \mathrm{~nm}\left(I_{340} / I_{440}\right)$ was found to increase with the amount of $\mathrm{Mg}^{2+}$ or $\mathrm{Ca}^{2+}$ added in the solution for both compounds BNI2 and BNI3 (Fig. 6, the insets). This provides an opportunity to calculate the concentration of a cation $\left(\left[\mathrm{M}^{n+}\right]\right)$ without the necessity to know exactly the concentration of a sensor according to the following equation: ${ }^{5}$

$$
\left[\mathrm{M}^{n+}\right]=K_{\mathrm{D}} \frac{R \quad R_{\min }}{R_{\max } \quad R},
$$

where $R$ is the ratio $I_{340} / I_{440}$ for the studied sample with an unknown $\mathrm{M}^{n+}$ content, $R_{\max }$ and $R_{\min }$ correspond to the ratios $I_{340} / I_{440}$ measured for the analyte bound and analyte free probes, respectively, and $K_{\mathrm{D}}$ is the complex dissociation constant. For instance, the ratio of emission intensities $R$ for the $6.5 \mu \mathrm{M}$ solution of BNI3 containing 2 equivalents of $\mathrm{Ca}^{2+}$ is 0.54 . Assuming that $R_{\min }$ and $R_{\max }$ values are as high as 0.15 and 0.92 (found from the titration curve (Fig. 6b)) and $K_{\mathrm{D}}=1 / K=1 / 10^{5.04} \mathrm{M}\left(\lg K=5.04\right.$ for $($ BNI3 $) \cdot \mathrm{Ca}^{2+}$, Table 1$)$, the equilibrium concentration of calcium cations in the solution is $\left[\mathrm{Ca}^{2+}\right]=9.4 \times 10^{-6} \mathrm{M}$. This value is very close to the one $(9.7 \times$ $10^{-6} \mathrm{M}$ ) obtained from the calculations of the solution composition using the SPECFIT/32 program.

\section{Conclusion}

Novel crown-containing naphthalimide dyads BNI2 and BNI3 were synthesized based on a convergent approach. The compounds were designed as ratiometric cation FRET chemosensors comprising the PET switching amido-naphthalimide fluorophore linked with the amino-naphthalimide fragment. Steady-state and time-resolved optical studies revealed that the resonance energy transfer operating between the photoactive units competes fairly well with the photoinduced electron transfer from the crown ether receptor and, thus, can be switched on by the presence of metal ions. As a result, a fluorescence enhancement of the acceptor amino-naphthalimide chromophore occurs upon complex formation with $\mathrm{Ca}^{2+}$ and $\mathrm{Mg}^{2+}$, a more pronounced effect being observed in the case of the aza-15-crown-5-containing derivative BNI3 where PET interaction was supposed to be stronger compared to BNI2.

In contrast to conventional "off-on" or "on-off" PET sensors with one fluorophore, our dyads open a way for ratiometric fluorescent detection of ions, because the FRET mediated "off-on" signal output obtained at $\lambda_{\text {ex }}=340 \mathrm{~nm}$ can be selfcalibrated with respect to the emission channel at $\lambda_{\mathrm{ex}}=440 \mathrm{~nm}$ entirely unresponsive to the presence of the analyte. Thus, the presented results have shown that compounds BNI2 and BNI3 can be of interest for the development of fluorescent ratiometric chemosensors for various kinds of cationic analysis. Due to versatile structural modification of the crown ether moiety, selective probes for various metal cations can be prepared. Related research is currently underway in our laboratory.

\section{Acknowledgements}

P.A.P. thanks RFBR project No. 14-03-31935 (design, synthesis, and characterization of the compounds). O.A.F. thanks RFBR project No. 15-03-04705 (complex formation studies). G.J. thanks the Région Aquitaine for financial support.

\section{References}

1 B. W. Van Der Meer, G. Coker and S. Y. S. Chen, Resonance Energy Transfer: Theory and Data, VCH, New York, 1994.

2 J. R. Lakowicz, Principles of fluorescent spectroscopy, Springer science + Business Media, LLC, Plenum Publishers, New York, 2006.

3 K. Kikuchi, H. Takakusa and T. Nagano, Trends Anal. Chem., 2004, 23, 407-415.

4 J. Fan, M. Hu, P. Zhan and X. Peng, Chem. Soc. Rev., 2013, 42, 29-43.

5 A. P. Demchenko, Lab Chip, 2005, 5, 1210-1223.

6 L. Tolosa, K. Nowaczyk and J. Lakowicz, An introduction to laser spectroscopy, Kluwer, New York, 2nd edn, 2002.

7 L. G. F. Patrick and A. Whiting, Dyes Pigm., 2002, 52, 137-143.

8 I. Grabchev and R. Betcheva, J. Photochem. Photobiol., A, 2001, 142, 73-78.

9 L. G. F. Patrick and A. Whiting, Dyes Pigm., 2002, 55, 123-132. 
10 E. Martin, R. Weigand and A. Pardo, J. Lumin., 1996, 68, 157-164.

11 W. W. Stewart, J. Am. Chem. Soc., 1981, 103, 7615-7620.

12 M. Sawa, T.-L. Hsu, T. Itoh, M. Sugiyama, S. R. Hanson, P. K. Vogt and C.-H. Wong, Proc. Natl. Acad. Sci. U. S. A., 2006, 103, 12371-12376.

13 H.-H. Lin, Y.-C. Chan, J.-W. Chen and C.-C. Chang, J. Mater. Chem., 2011, 21, 3170-3177.

14 S. Banerjee, E. B. Veale, C. M. Phelan, S. A. Murphy, G. M. Tocci, L. J. Gillespie, D. O. Frimannsson, J. M. Kelly and T. Gunnlaugsson, Chem. Soc. Rev., 2013, 42, 1601-1618.

15 W. Zhu, M. Hu, R. Yao and H. Tian, J. Photochem. Photobiol., A, 2003, 154, 169-177.

16 G. Tu, Q. Zhou, Y. Cheng, Y. Geng, L. Wang, D. Ma, X. Jing and F. Wang, Synth. Met., 2005, 152, 233-236.

17 C. Coya, R. Blanco, R. Juárez, R. Gómez, R. Martínez, A. de Andrés, Á. L. Álvarez, C. Zaldo, M. M. Ramos, A. de la Peña, C. Seoane and J. L. Segura, Eur. Polym. J., 2010, 46, 1778-1789.

18 L. Song, E. A. Jares-Erijman and T. M. Jovin, J. Photochem. Photobiol., A, 2002, 150, 177-185.

19 X. Meng, W. Zhu, Q. Zhang, Y. Feng, W. Tan and H. Tian, J. Phys. Chem. B, 2008, 112, 15636-15645.

20 O. A. Fedorova, P. A. Panchenko, Y. V. Fedorov, F. G. Erko, J. Berthet and S. Delbaere, J. Photochem. Photobiol., A, 2015, 303-304, 28-35.

21 I. Grabchev and I. Moneva, J. Polym. Sci., 1999, 74, 151-157.

22 Y. Zhang, W. Zhu, W. Wang, H. Tian, J. Su and W. Wang, J. Mater. Chem., 2002, 12, 1294-1300.

23 P. A. Panchenko, O. A. Fedorova and Y. V. Fedorov, Russ. Chem. Rev., 2014, 83, 155-182.

24 R. M. Duke, E. B. Veale, F. M. Pfeffer, P. E. Kruger and T. Gunnlaugsson, Chem. Soc. Rev., 2010, 39, 3936-3953.

25 N. I. Georgiev, V. B. Bojinov and P. S. Nikolov, Dyes Pigm., 2009, 81, 18-26.

26 N. I. Georgiev, V. B. Bojinov and N. Marinova, Sens. Actuators, $B, 2010,150,655-666$.

27 N. I. Georgiev, A. M. Asiri, A. H. Qusti, K. A. Alamry and V. B. Bojinov, Sens. Actuators, B, 2014, 190, 185-198.

28 X. Zhou, F. Su, H. Lu, P. Senechal-Willis, Y. Tian, R. H. Johnson and D. R. Meldrum, Biomaterials, 2012, 33, 171-180.

29 B. H. Shankar and D. Ramaiah, J. Phys. Chem. B, 2011, 115, 13292-13299.

30 V. S. Jisha, A. J. Thomas and D. Ramaiah, J. Org. Chem., 2009, 74, 6667-6673.
31 Z. Zhou, M. Yu, H. Yang, K. Huang, F. Li, T. Yi and C. Huang, Chem. Commun., 2008, 3387-3389.

32 V. B. Bojinov, A. I. Venkova and N. I. Georgiev, Sens. Actuators, B, 2009, 143, 42-49.

33 Q. Wang, C. Li, Y. Zou, H. Wang, T. Yi and C. Huang, Org. Biomol. Chem., 2012, 10, 6740-6746.

34 P. Mahato, S. Saha, E. Suresh, R. D. Liddo, P. P. Parnigotto, M. T. Conconi, M. K. Kesharwani, B. Ganguly and A. Das, Inorg. Chem., 2012, 51, 1769-1777.

35 Y. Liu, X. Lv, Y. Zhao, M. Chen, J. Liu, P. Wang and W. Guo, Dyes Pigm., 2012, 92, 909-915.

36 J. Fan, C. Lin, H. Li, P. Zhan, J. Wang, S. Cui, M. Hu, G. Cheng and X. Peng, Dyes Pigm., 2013, 99, 620-626.

37 J. Fan, P. Zhan, M. Hu, W. Sun, J. Tang, J. Wang, S. Sun, F. Song and X. Peng, Org. Lett., 2013, 15, 492-495.

38 C.-Y. Li, Y. Zhou, Y.-F. Li, C.-X. Zou and X.-F. Kong, Sens. Actuators, B, 2013, 186, 360-366.

39 N. I. Georgiev, M. D. Dimitrova, A. M. Asiri, K. A. Alamry and V. B. Bojinov, Dyes Pigm., 2015, 115, 172-180.

40 K. A. Alamry, N. I. Georgiev, S. A. El-Daly, L. A. Taib and V. B. Bojinov, J. Lumin., 2015, 158, 50-59.

41 P. A. Panchenko, Y. V. Fedorov, V. P. Perevalov, G. Jonusauskas and O. A. Fedorova, J. Phys. Chem. A, 2010, 114, 4118-4122.

42 P. A. Panchenko, Y. V. Fedorov, O. A. Fedorova, B. A. Izmailov, V. A. Vasnev, V. V. Istratov, E. A. Makeeva, M. N. Rumyantseva and A. M. Gaskov, Mendeelev Commun., 2011, 21, 12-14.

43 P. A. Panchenko, Y. V. Fedorov, O. A. Fedorova and G. Jonusauskas, Dyes Pigm., 2013, 98, 347-357.

44 P. A. Panchenko, Y. V. Fedorov, O. A. Fedorova, V. P. Perevalov and G. Jonusauskas, Russ. Chem. Bull., 2009, 58, 1233-1240.

45 S. Nad, M. Kumbhakar and H. Pal, J. Phys. Chem. A, 2003, 107, 4808-4816.

46 C. L. Renschler and L. A. Harrah, Anal. Chem., 1983, 55, 798-800.

47 K. A. Connors, Binding constants: the measurement of molecular complex stability, John Wiley \& Sons, New York, 1987.

48 M. T. Beck and I. Nagypál, Chemistry of complex equilibria, John Wiley \& Sons, New York, 1990.

49 D. L. Perry, Handbook of Inorganic Compounds, CRC Press, Boca Raton, 2011.

50 P. O'Neill, S. Steenken and D. Schulte-Frohlinde, J. Phys. Chem., 1975, 79, 2773-2779.

51 T. Shida, Y. Nosaka and T. Kato, J. Phys. Chem., 1978, 82, 695-698. 